\title{
The role of the $\alpha 4$ integrin-paxillin interaction in regulating leukocyte trafficking
}

\author{
David M. Rose \\ Department of Medicine \\ University of California/VA Medical Center \\ San Diego, CA, USA \\ Correspondence: Tel, 858-552-8585 ext. 5378; \\ Fax, 858-552-7425; E-mail, drose@vapop.ucsd.edu
}

Abbreviations: Arf, ADP-ribosylation-factor; FAK, focal adhesion kinase; GIT, G protein-coupled receptor kinase interactor; PKA, protein kinase $\mathrm{A}$; $\mathrm{PKL}$, paxillin kinase linker; $\mathrm{PML}$, progressive multifocal leukoencephalophathy; PTP-PEST, protein tyrosine phosphatase-PEST; Pyk2, proline-rich tyrosine kinase

\begin{abstract}
The movement of leukocytes from the blood into peripheral tissues is a central feature of immune surveillance, but also contributes to the pathogenesis of inflammatory and autoimmune diseases. Integrins are a family of adhesion and signaling molecules made up of paired $\alpha$ and $\beta$ subunits, and the integrin $\alpha 4 \beta 1$ plays a prominent role in the trafficking of mononuclear leukocytes. We have previously described the direct interaction of the signaling adaptor molecule paxillin with the cytoplasmic domain of the $\alpha 4$ integrin subunit. This interaction is critical for $\alpha 4 \beta 1$ integrin dependent cell adhesion under shear flow conditions as it provides a needed connection to the actin cytoskeleton. Furthermore, the $\alpha 4$-paxillin interaction is required for effective $\alpha 4 \beta 1$ dependent leukocyte migration and does so through the temporal and spatial regulation of the small GTPase Rac. These findings make the $\alpha 4$-paxillin interaction a poten tially attractive therapeutic target in controlling leukocyte trafficking.
\end{abstract}

Keywords: $\alpha 4$ integrins; autoimmune diseases; leukocyte trafficking; paxillin

\section{Introduction}

Leukocyte trafficking is the process by which circulating leukocytes in blood leave and enter the peripheral tissues. This event is critical for both immune surveillance and inflammation. Over the past
20 yrs a paradigm has developed in which the control of leukocyte trafficking is modeled after the mail zip code system; namely, specific combinations of adhesion and chemoattractant molecules expressed both on leukocytes and the vascular endothelium direct the temporal and spatial recruitment of leukocytes around the body (Butcher, 1991; Springer, 1994). A comprehensive review of leukocyte trafficking is beyond the scope of this review, but the reader is directed to recent reviews (Butcher, 1991; Springer, 1994; Luster et al., 2005). Instead, this review focuses on recent work defining the role of the cell adhesion molecule $\alpha 4 \beta 1$ on leukocyte trafficking and in particular the importance of the interaction of the signaling adapter molecule paxillin with the $\alpha 4$ subunit in regulating $\alpha 4 \beta 1$-dependent functions.

In general, the movement of leukocytes from the blood into tissues involves three sequential steps (Butcher, 1991; Springer, 1994). First, leukocytes make transient tethering adhesions with the vascular endothelium, which under shear flow conditions results in leukocyte rolling along the endothelial surface. Second, should the leukocyte encounter an appropriate stimulus, it undergoes activation and establishes firm adhesion to the endothelium. Third, the leukocyte migrates across the endothelium into the underlying tissue. Integrins are a family of cell surface adhesion and signaling molecules made up of non-covalently associated $\alpha$ and $\beta$ subunits that play a critical role in leukocyte trafficking (Luster et al., 2005). The integrin $\alpha 4 \beta 1$, the focus of this review, is rather unique among integrins as it can function in all three steps of leukocyte trafficking (Berlin et al., 1995; Rose et al., 2002). $\alpha 4 \beta 1$ can be found on virtually all circulating mononuclear leukocytes, but is not typically seen on unactivated neutrophils (Rose et al., 2002). Integrin subunits have relatively short cytoplasmic domains, but they can associate with numerous proteins allowing linkage to the cytoskeleton and formation of signaling complexes (Liu et al., 2000a). Both interactions are critical to integrin-dependent cell adhesion and migration. One such integrin-protein interaction is the rather unique association of the $\alpha 4$ integrin subunit with the signaling adaptor molecule paxillin (Liu et al., 1999).

Paxillin is a wildly expressed $68 \mathrm{kD}$ cytoplasmic protein that can be found associated with cell adhesion complexes (Brown and Turner, 2004). It was 
one of the first signaling adaptor molecules described; it has no intrinsic kinase or phosphatase activity yet it can scaffold a number of signaling molecules such as kinases, other adaptor proteins, and nucleotide exchange factors as well as binding cytoskeleton proteins (Figure 1). The binding of these signaling molecules to paxillin is largely mediated by the presence of 5 leucine-rich LD domains in the $\mathrm{N}$-terminus and 4 zinc finger LIM domains in paxillin's C-terminus (Brown and Turner, 2004). The $\alpha 4$ integrin binding site on paxillin has been partially localized to a 100 amino acid stretch encompassing LD3 and LD4 (Liu et al., 2002). The region of the cytoplasmic domain of $\alpha 4$ integrin that mediates paxillin binding rests in the nine amino acids between Glu983 and Tyr991 (Liu et al., 2000b). Mutation of this Tyr991 to an Ala disrupts the $\alpha 4$ paxillin interaction (Liu et al., 1999). Further, a serine at position 988 can be reversibly phosphorylated in a PKA-dependent fashion to regulate paxillin binding to $\alpha 4$ (Han et al., 2003). The temporal and spatial regulation of this serine phosphorylation and associated paxillin binding to $\alpha 4$ is critical in effective cell migration (discussed below) (Goldfinger et al., 2003).

\section{The $\alpha 4$ integrin-paxillin interaction in cell adhesion under shear flow}

The $\alpha 4 \beta 1$ integrin is one of the few integrins that can support tethering and rolling of leukocytes under shear flow conditions, a process largely associated with the selectin family of adhesion molecules (Alon, et al., 1995; Rose et al., 2002; Rosen, 2004). While initial studies with a truncation of the $\alpha 4$ cytoplasmic domain suggested this region was not involved in regulating tethering interactions, it has become clear that such a truncation may have secondarily altered $\beta 1$ integrin function masking the role of the $\alpha 4$ cytoplasmic domain in supporting tethering adhesion under flow conditions (Kassner et al., 1995). We recently have demonstrated that the $\alpha 4$-paxillin interaction is critical to establishing tethering adhesion, and that adhesion strength is mediated thru connections with the actin cytoskeleton (Alon et al., 2005). In particular, paxillin binding to the cytoskeletal protein talin is central in linking the $\alpha 4$ integrin with the actin cytoskeleton (Alon et al., 2005). Jurkat cells expressing $\alpha 4 Y 991 \mathrm{~A}$ with disrupted paxillin binding to $\alpha 4$ had reduced tethering bond strength associated with reduced talin in the $\alpha 4$ integrin adhesion complex (Alon et al., 2005). Furthermore, knocking-down talin expression in Jurkat cells expressing $\alpha 4$ WT integrin impaired $\alpha 4$-dependent tethering under shear flow (Alon et al.,
2005). Talin has been established to be a key molecule regulating integrin affinity for ligands (a process termed inside-out signaling) (Tadokoro et al., 2003). However, the role of talin in $\alpha 4$-paxillin dependent tethering does not appear to involve $\alpha 4$ integrin affinity modulation (Rose et al., 2003). First, cells expressing $\alpha 4$ with disrupted paxillin binding had no defect in $\alpha 4 \beta 1$ integrin affinity for its ligand VCAM-1 (Rose et al., 2003). Second, ligand-induced conformation changes in $\alpha 4 \beta 1$ were not altered by disrupting the $\alpha 4$-paxillin interaction nor were conformational changes altered by knocking down talin levels (Alon et al., 2005). Thus, the role of the $\alpha 4$-paxillin interaction in mediating leukocyte tethering is through establishment of associations with the cytoskeleton to strengthen adhesion under shear flow conditions.

The $\alpha 4$-paxillin interaction is also important in establishing firm cell adhesion under shear stress conditions (Alon et al., 2005). This is in contrast to static cell adhesion, which is unaltered by disrupting the $\alpha 4$-paxillin interaction (Rose et al., 2003). Again, the mechanism underlying this cell adhesion strengthening under shear flow conditions is associated with $\alpha 4$-paxillin-dependent connections with the actin cytoskeleton. Further details on the establishment of these connections between the $\alpha 4$ integrin and the actin cytoskeleton are lacking. The a4-paxillin interaction is known to regulate focal adhesion dynamics through FAK and Pyk-2 activation as well as activation of small GTPases such as Rac, CDC42, and Rho (Rose et al., 2003). But these signaling molecules do not appear to be involved in the early events in adhesion under shear stress (Alon et al., 2005).

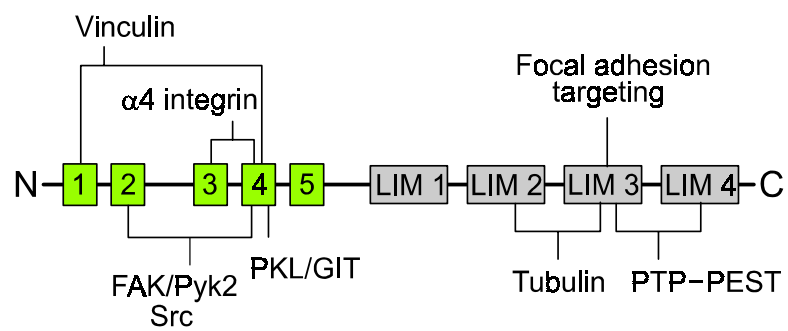

Figure 1. Paxillin associated proteins. Paxillin is comprised of 5 LD motifs in its $\mathrm{N}$-terminus and 4 LIM domains in its $\mathrm{C}$-terminus. These regions serve as primary docking sites for a number of signaling and cytoskeletal proteins (not all of which are shown in this figure). Binding partners include kinases such as FAK, Pyk2 and Src as well as phosphatases such as PTP-PEST. Cytoskeletal proteins binding to paxillin include vinculin and tubulin. 


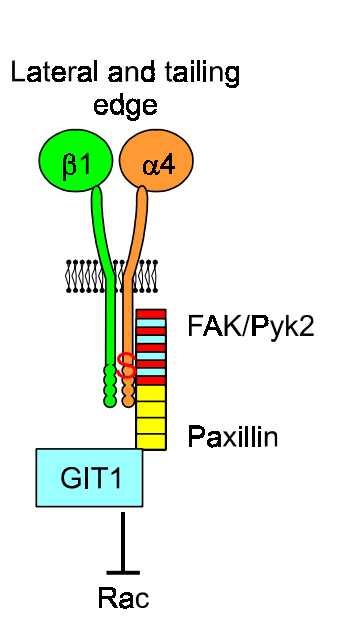

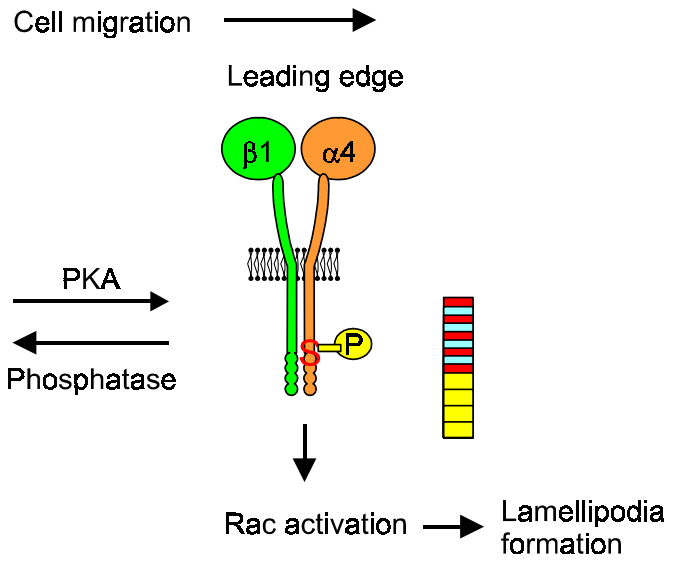

Figure 2. Dynamic regulation of paxillin binding to the $\alpha 4$ integrin subunit by serine phosphorylation of $\alpha 4$ regulates spacial activation of the small GTPase Rac in migrating cells. Paxillin associates with the $\alpha 4$ integrin when the serine at position 988 of the $\alpha 4$ cytoplasmic domain is unphosphorylated. This $\alpha 4$-paxillin complex recruits the GTPase activating protein GIT1, which subsequently inhibits Arf6 activation and ultimately inhibits Rac activation. Inhibition of Rac prevents lamellipodia formation at the lateral and tailing edge of a migrating cell. Phosphorylation of $\alpha 4$ serine 988 inhibits paxillin binding and removes the suppression of Rac activation, ultimately resulting in lamellipodia formation at the leading edge of the migrating cell.

\section{The $\alpha 4$ integrin-paxillin interaction in regulating cell migration}

After a leukocyte has firmly arrested on the vascular endothelium, it then migrates laterally to the junction of two or more endothelial cells, where it may migrate in between these cells into the underlying tissue. $\alpha 4$ integrins play roles in both these migratory steps of leukocyte trafficking (Weber and Springer, 1998). Recently, much has been learned about the coordinated signaling involved in cell migration, and the role of the $\alpha 4$-paxillin interaction in $\alpha 4$ integrin-dependent cell migration. Cell migration involves a dynamic regulation of cell adhesion in which new adhesions are formed at the leading edge of the cell where lamellipodia are sent out as new projections and adhesions are released from the trailing edge of the cell as the body of the cell is propelled forward (Ridley et al., 2003).

Spatial and temporal regulation of the small GTPase Rac is central to effective cell migration such that Rac is activated at the leading edge of the cell promoting lamellipodia formation, while Rac activation is inhibited at the lateral and trailing edge of the cell (Nobes and Hall, 1999). The $\alpha 4$ integrin subunit is dephosphorylated at Ser988 at the lateral and trailing edge of a migrating cell, which is associated with paxillin binding to $\alpha 4$ at these locations (Goldfinger et al., 2003) (Figure 2). At these sites, paxillin binding to $\alpha 4$ blocks lamellipodia formation by inhibiting Rac activation (Nishiya et al., 2005). This occurs as paxillin recruits the GTPase activating protein GIT1 (Nishiya et al., 2005). GIT1 inhibits Arf6, another member of the Ras small GTPase family. The inhibition of Arf6 subsequently inhibits the activation of Rac by yet unknown mechanisms.

In contrast, at the leading edge of the migrating cell, the $\alpha 4$ subunit is phosphorylated resulting in disruption of the $\alpha 4$-paxillin interaction (Goldfinger et al., 2003) (Figure 2). This results in the removal of the $\alpha 4$-paxillin-dependent inhibition of Rac activation at this site (Nishiya et al., 2005). It has recently been proposed that the ultimate activation of Rac by $\alpha 4$ integrins proceeds through a pathway dependent on Src activation (Hsia et al., 2005). Src activation subsequently leads to Rac activation through the activation of the Rac exchange factor DOCK180.

In addition to functioning as a direct adhesion molecule mediating cell migration, $\alpha 4$ integrins can also function as a signaling molecule augmenting cell migration mediated by other integrins in a transdominant fashion (Rose et al., 2001). $\alpha 4 \beta 1$ integrins can stimulate $\alpha \mathrm{L} \beta 2$ dependent cell migration, and this requires the $\alpha 4-$ paxillin interaction (Rose et al., 2001; Rose et al., 2003). This transdominant regulation is mediated through the $\alpha 4$-paxillin dependent activation of the nonreceptor tyrosine kinases FAK and Pyk-2 (Rose et al., 2003). Furthermore, the affinity of $\alpha 4 \beta 1$ also plays a role in stimulating $\alpha L \beta 2$-dependent cell migration as $\alpha 4 \beta 1$ in the high affinity state favors interactions with VCAM-1 to drive $\beta 2$ integrin-dependent migration (Rose et al., 2001). In addition, high affinity $\alpha 4 \beta 1$ preferentially associates with paxillin (Hyduk et al., 2004). Thus, while paxillin does not regulate the affinity of $\alpha 4 \beta 1$, its association with high affinity $\alpha 4 \beta 1$ contributes to the cross-talk with $\beta 2$ integrin to drive cell migration.

\section{Therapeutic potential of targeting the $\alpha 4$ integrin-paxillin interaction}

Given the role of the $\alpha 4$ integrins in trafficking of leukocytes, they have been an attractive target in the treatment of numerous chronic inflammatory and 
autoimmune diseases such as multiple sclerosis, inflammatory bowel disease, rheumatoid arthritis, and atherosclerosis (Gonzalez-Amaro, et al., 2005). Indeed, inhibition of $\alpha 4$ integrins with function blocking antibodies and small molecule inhibitors has been effective in attenuating many mouse models of these diseases (Gonzalez-Amaro, et al., 2005). Further, a humanized function-blocking antibody to $\alpha 4$ has been shown to be effective in clinical trials of patients with multiple sclerosis and inflammatory bowel disease (Miller et al., 2003; Ghosh et al., 2003). However, given the role of $\alpha 4$ integrins in normal physiological functions such as hematopoiesis and immune surveillance, the risk of side-effects of $\alpha 4$ integrin blockage has been a concern. The development of progressive multifocal leukoencephalophathy $(\mathrm{PML})$ in three patients treated with anti- $\alpha 4$ antibodies has highlighted this concern as PML occurs most commonly due to reemergence of a latent $\mathrm{JC}$ virus infection in the central nervous system in immune suppressed individuals (Yousry et al., 2006). Consequently, a more selective targeting of $\alpha 4$ integrins to inhibit leukocyte trafficking while sparing its function in normal physiology may be therapeutically beneficial. To this aim, targeting the $\alpha 4$-paxillin interaction may be one such possibility. A screening of a combinatorial library has successfully identified a small molecule inhibitor of the $\alpha 4$-paxillin interaction in vitro that inhibits cell migration mediated by $\alpha 4 \beta 1$ (Ambroise et al., 2002).

Further support of the proof of principle of targeting the $\alpha 4$-paxillin interaction comes from recent studies of a novel knock-in mouse in which the Y991A mutation that disrupts the $\alpha 4$-paxillin interaction has been introduced into the mouse genome (Feral et al., 2006). Such mice are born viable with no defect in placentation or heart development, which occurred in mice with complete gene ablation of $\alpha 4$ integrin (Feral et al., 2006). Furthermore, these mice have no defect in hematopoiesis or immune system development. But importantly, they show impairment in the recruitment of mononuclear leukocytes to a site of inflammation (Feral et al., 2006). Thus, targeting the $\alpha 4$-paxillin interaction may have the potential to impair leukocyte trafficking associated with chronic inflammatory diseases, while sparing $\alpha 4$ integrin function in normal physiology.

\section{References}

Alon R, Feigelson SW, Manevich E, Rose DM, Schmitz J, Overby DR, Winter E, Grabovsky V, Shinder V, Matthews BD, Sokolovsky-Eisenberg M, Ingber DE, Benoit M, Ginsberg MH. Alpha4beta1-dependent adhesion strengthening under mechanical strain is regulated by paxillin association with the alpha4-cytoplasmic domain. J Cell Biol 2005;171:1073-84
Alon R, Kassner PD, Carr MW, Finger EB, Hemler ME, Springer TA. The integrin VLA-4 supports tethering and rolling in flow on VCAM-1. J Cell Biol 1995;128:1243-53

Ambroise Y, Yaspan B, Ginsberg MH, Boger DL. Inhibitors of cell migration that inhibit intracellular paxillin/alpha4 binding: a well-documented use of positional scanning libraries. Chem Biol 2002;9:1219-26

Berlin C, Bargatze RF, Campbell JJ, von Andrian UH, Szabo MC, Hasslen SR, Nelson RD, Berg EL, Erlandsen SL, Butcher EC. alpha 4 integrins mediate lymphocyte attachment and rolling under physiologic flow. Cell 1995;30:413-22

Brown MC, Turner CE. Paxillin: adapting to change. Physiol Rev 2004;84:1315-39

Butcher EC. Leukocyte-endothelial cell recognition: three (or more) steps to specificity and diversity. Cell 1991;67:1033-6

Feral CC, Rose DM, Han J, Fox N, Silverman GJ, Kaushansky $\mathrm{K}$, Ginsberg MH. Blocking the alpha 4 integrin-paxillin interaction selectively impairs mononuclear leukocyte recruitment to an inflammatory site. J Clin Invest 2006;116:715-23

Ghosh S, Goldin E, Gordon FH, Malchow HA, Rask-Madsen J, Rutgeerts P, Vyhnalek P, Zadorova Z, Palmer T, Donoghue $S$; Natalizumab Pan-European Study Group. Natalizumab for active Crohn's disease. N Engl J Med 2003;348:24-32

Goldfinger LE, Han J, Kiosses WB, Howe AK, Ginsberg MH. Spatial restriction of alpha4 integrin phosphorylation regulates lamellipodial stability and alpha4beta1-dependent migration. J Cell Biol 2003;162:731-41

Gonzalez-Amaro R, Mittlebrunn M, Sanchez-Madrid F. Therapeutic anti-integrin (alpha4 and alphaL) monoclonal antibodies: two-edged swords? Immunology 2005;116:289-96

Han J, Rose DM, Woodside DG, Goldfinger LE, Ginsberg MH. Integrin alpha 4 beta 1-dependent $T$ cell migration requires both phosphorylation and dephosphorylation of the alpha 4 cytoplasmic domain to regulate the reversible binding of paxillin. J Biol Chem 2003;278:34845-53

Hsia DA, Lim ST, Bernard-Trifilo JA, Mitra SK, Tanaka S, den Hertog J, Streblow DN, llic D, Ginsberg MH, Schlaepfer DD. Integrin alpha4beta1 promotes focal adhesion kinase-independent cell motility via alpha4 cytoplasmic domain-specific activation of c-Src. Mol Cell Biol 2005;25:9700-12

Hyduk SJ, Oh J, Xiao H, Chen M, Cybulsky MI. Paxillin selectively associates with constitutive and chemoattractant-induced high-affinty alpha4beta1 integrins: implications for integrin signaling. Blood 2004;104:2818-24

Kassner PD, Alon R, Springer TA, Hemler ME. Specialized functional properties of the integrin alpha 4 cytoplasmic domain. Mol Biol Cell 1995;6:661-74

Liu S, Calderwood DA, Ginsberg MH. Integrin cytoplasmic domain-binding proteins. J Cell Sci 2000a;113:3563-71

Liu S, Ginsberg MH. Paxillin binding to a conserved sequence motif in the alpha 4 integrin cytoplasmic domain. J Biol Chem 2000b;274:22736-42

Liu S, Kiosses WB, Rose DM, Slepak M, Salgia R, Griffin JD, Turner CE, Schwartz MA, Ginsberg MH. A fragment of paxillin binds the alpha 4 integrin cytoplasmic domain (tail) and se- 
lectively inhibits alpha 4-mediated cell migration. J Biol Chem 2002;277:20887-94

Liu S, Thomas SM, Woodside DG, Rose DM, Kiosses WB, Pfaff M, Ginsberg MH. Binding of paxillin to alpha4 integrins modifies integrin-dependent biological responses. Nature 1999;402:676-81

Luster AD, Alon R, von Andrian UH. Immune cell migration in inflammation: present and future therapeutic targets. Nat Immunol 2005;6:1182-90

Miller DH, Khan OA, Sheremata WA, Blumhardt LD, Rice GP, Libonati MA, Willmer-Hulme AJ, Dalton CM, Miszkiel KA, O'Connor PW; International Natalizumab Multiple Sclerosis Trial Group. A controlled trial of natalizumab for relapsing multiple sclerosis. N Engl J Med 2003;348:15-23

Nishiya N, Kiosses WB, Han J, Ginsberg MH. An alpha4 integrin-paxillin-Arf-GAP complex restricts Rac activation to the leading of migrating cells. Nat Cell Biol 2005;7:343-52

Nobes CD, Hall A. Rho GTPases control plarity, protrusion, and adhesion during cell movement. J Cell Biol 1999; 144:1235-44

Ridley AJ, Schwartz MA, Burridge K, Firtel RA, Ginsberg MH, Borisy G, Parsons JT, Horwitz AR. Cell migration: integrating signals from front to back. Science 2003;302:1704-9

Rose DM, Grabovsky V, Alon R, Ginsberg MH. The affinity of integrin alpha(4)beta(1) governs lymphocyte migration. J Immunol 2001;167:2824-30
Rose DM, Han J, Ginsberg MH. Alpha4 integrins and the immune response. Immunol Rev 2002;186:118-24

Rose DM, Liu S, Woodside DG, Han J, Schlaepfer DD, Ginsberg MH. Paxillin binding to the alpha 4 integrin subunit stimulates LFA-1 (integrin alpha $L$ beta 2 )-dependent $T$ cell migration by augmenting the activation of focal adhesion kinase/proline-rich tyrosine kinase-2. J Immunol 2003;170: 5912-8

Rosen SD. Ligands for L-selectin: homing, inflammation, and beyond. Annu Rev Immunol 2004;22:129-56

Springer TA. Traffic signals for lymphocyte recirculation and leukocyte emigration: the multistep paradigm. Cell 1994;76: 301-14

Tadokoro S, Shattil SJ, Eto K, Tai V, Liddington RC, de Pereda JM, Ginsberg MH, Calderwood DA. Talin binding to integrin beta tails: a final common step in integrin activation. Science 2003;302:103-6

Weber C, Springer TA. Interaction of very late antigen-4 with VCAM-1 supports transendothelial chemotaxis of monocytes by facilitating lateral migration. J Immunol 1998;161:6825-34

Yousry TA, Major EO, Ryschkewitsch C, Fahle G, Fischer S, Hou J, Curfman B, Miszkiel K, Mueller-Lenke N, Sanchez E, Barkhof F, Radue EW, Jager HR, Clifford DB. Evaluation of patients treated with natalizumab for progressive multifocal leukoencephalopathy. N Engl J Med 2006;354:924-33 